\title{
Fluorene-Based Rib Waveguides with Optimized Geometry for Long-Term Amplified Spontaneous Emission Stability
}

\author{
Gonzalo Del Pozo', Noureddine Bennis², Xabier Quintana ${ }^{3}$, Jose Manuel Otón ${ }^{3}$, Jinyi Lin ${ }^{4}$, Qi Wei ${ }^{4}$ \\ Ruidong $\mathrm{Xia}^{4^{*}}$, Ramon Bernardo ${ }^{1}$, Daniel Granados ${ }^{1}$ and Juan Cabanillas-Gonzalez ${ }^{{ }^{*}}$
}

1. Madrid Institute for Advanced Studies - IMDEA Nanociencia, Calle Faraday 9, Ciudad Universitaria de Cantoblanco 28049, Spain.

2. Institute of Applied Physics, Military University of Technology, Kaliskiego 2, 00-908 Warsaw, Poland.

3. CEMDATIC, Escuela Técnica Superior de Ingenieros de Telecomunicación, Universidad Politécnica de Madrid, Av. Complutense 30, 28040 Madrid, Spain.

4. Key Laboratory for Organic Electronics and Information Displays \& Institute of Advanced Materials, National Jiangsu Synergistic Innovation Center for Advanced Materials (SICAM), Nanjing University of Posts and Telecommunications, 9 Wenyuan Road, Nanjing 210046, P.R., China.

*Correspondence to: Juan Cabanillas-Gonzalez (E-mail: juan.cabanillas@imdea.org), Ruidong Xia (E-mail: ruidong.xia@gmail.com)

ABSTRACT: Amongst the different optoelectronic applications of conjugated polymers, waveguide amplifiers and optically-pumped lasers are those requiring larger photochemical stability, owing to the large irradiation conditions under operation. In this context, suitable waveguide optimization enabling the reduction of amplified spontaneous emission (ASE) threshold values appears as important as synthetic chemistry protocols to promote polymer robustness against photo-oxidation. In this work we develop rib waveguides with different geometries based on four different fluorene-based compounds and assess the influence of rib confinement on ASE properties. We observe ASE threshold values as low as $8.910^{-4} \mathrm{mJcm}^{-2}$, being among the lowest ASE threshold values reported so far on blue emitting polymer / oligomer waveguides. We demonstrate that the increase in ASE efficiency on some of these rib waveguides leads to a five-fold increase in operation lifetime respect to spincoated slab waveguides, thus confirming the impact of waveguide geometry on ASE operation stability.

KEYWORDS: organic lasers, conjugated polymers, fluorescence, photolithography, oligofluorenes

\section{INTRODUCTION}

Conjugated polymers are materials which exhibit outstanding optical gain properties.[1-3] Processed as thin films they behave as slab waveguides, their large stimulated emission cross-section values and waveguiding properties enabling amplified spontaneous emission at very low pump thresholds, compared with organic dyes.[4-5] These assets together with their semiconducting charge transport behavior open up prospects for the future development of polymer-based electrically pumped lasers.[6-7] Among conjugated polymers, poly(9,9-di-octylfluorene) (PFO) is one of the most representative optical amplifying polymers.[810] Its notable properties as optical gain 
medium arise from a combination of efficient solid state blue emission[11] (c.a. 40\%) and excellent thin film optical quality which manifests into reduced scattering-induced losses and large waveguide performance.[12]
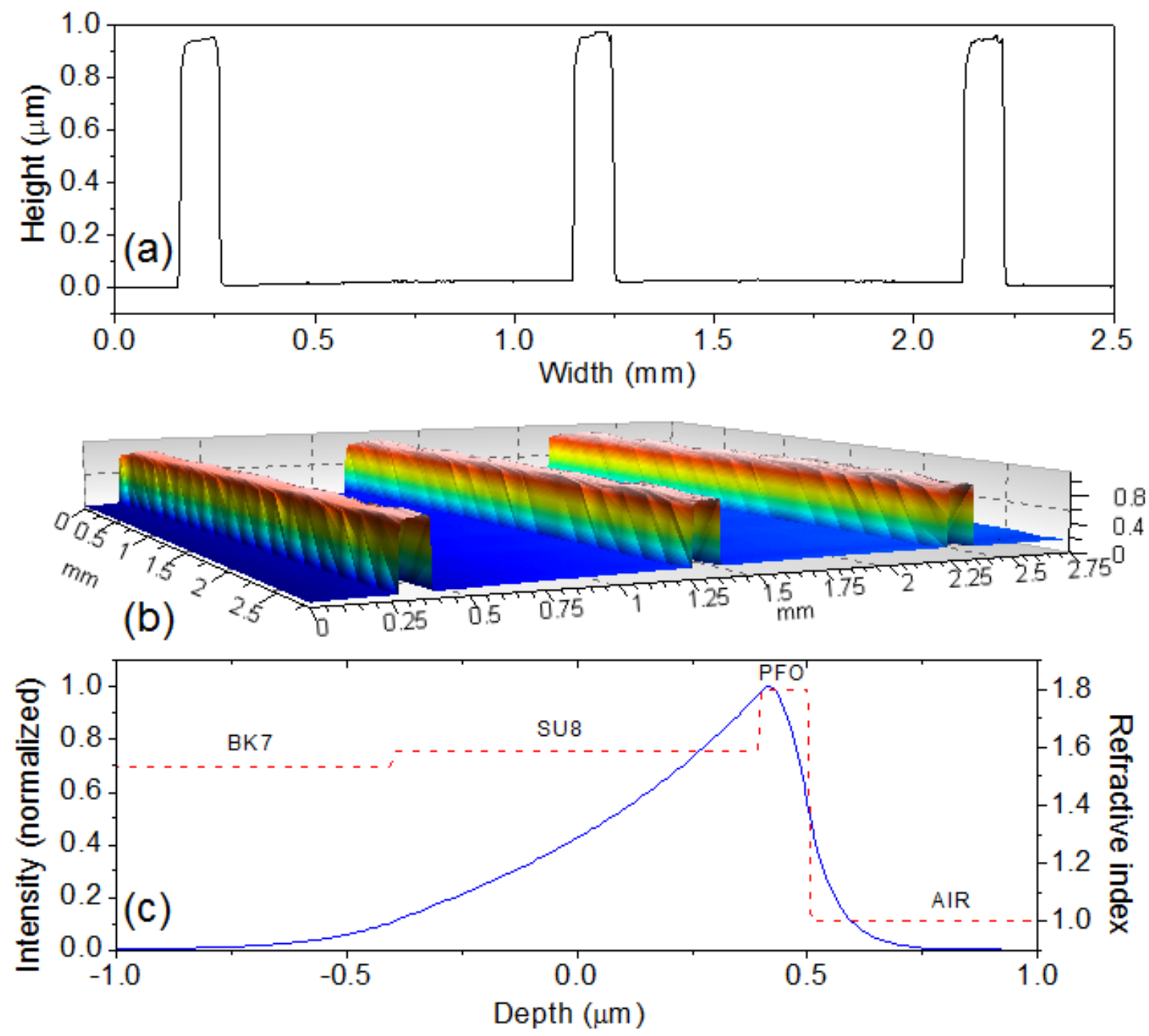

FIGURE 1. (a) Profile of the $100 \mu \mathrm{m}$ waveguide. (b) 3D reconstruction of the waveguide, (c) in depth intensity distribution of the fundamental propagating mode.

Hitherto, its operation lifetime is compromised by its low emission stability associated to interchain aggregation-induced energy transfer to fluorenone defects formed upon photooxidation,[13] a behavior which is particularly pronounced under intense UV irradiation. This problem is partially circumvented by reducing inter-chain aggregation, a solution which has been addressed with supramolecular chemical strategies, such as backbone encapsulation with cyclodextrin rings,[14-15] or strategies involving the design of side-chain substituents which interfere with backbone $\pi-\pi$ stacking.[16-17] It has been shown in oligofluorenes that the replacement of alkyl- side-chains by di-aryl groups has led to strong changes in molecular packing inferred from the optical spectra.[18] In line with this second strategy, some of us have already reported on $\mathrm{H}$-shape fluorene-based oligomers bearing one terfluorene arm with flexible alkyl chains and a carbazole-bridged rigid and highly non planar diarylfluorene arm.[19] The large steric hindrance induced by the diaryl-fluorene substitution was manifested 
on enhanced emission stability and absence of fluorenone emission upon deliberate aging. Additionally, enhanced thermodynamical stability was observed, as manifested by large glassy morphologies associated (Tg $250 \stackrel{\circ}{\circ}$ ) and unaltered ASE behavior upon air annealing

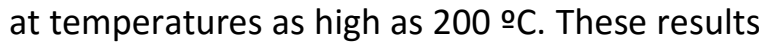
suggest that $\mathrm{H}$-shape oligofluorenes are promising compounds for applications involving optical amplification. In this work we develop rib waveguides based on these compounds as well as other three fluorene-based polymers with different side-chain substituents. Optical confinement induced by the rib leads to lowering of ASE thresholds by one order of magnitude respect to spincoated films. The width of the rib is found to have a strong influence on ASE threshold. Our results highlight the impact of chemical structure and waveguide geometry on long term stability of fluorenebased waveguides.

\section{EXPERIMENTAL}

Rib waveguides were fabricated by spincoating an epoxy-based negative SU8 photoresist (Gersteltec $\mathrm{GmbH}$ ), previously heated at $65 \stackrel{\circ}{ } \mathrm{C}$, at $4500 \mathrm{~nm} \mathrm{rpm}$ on top of a $10 \mathrm{~nm} \mathrm{SiO} 2$ coated glass substrate. For this purpose, glass substrates were previously cleaned in several ultrasonic baths and dried in a hot stage, before being coated with $\mathrm{SiO}_{2}$ by electro gun evaporation method at a fixed deposition rate of $1 \AA s^{-1}$. The $\mathrm{SiO}_{2}$ coating was deposited in order to improve the adhesion of SU8 to the substrate. The resulting SU8 layer (c.a. $800 \mathrm{~nm}$ thick) was then subsequently baked in two stages: $65^{\circ} \mathrm{C}$ for 5 minutes and $90 \circ \mathrm{C}$ for 10 minutes. Rib geometries were developed using the standard photolithography process upon UV exposition of SU8 through a shadow mask (365 $\mathrm{nm}, 100 \mathrm{mWcm}^{-2}$ for $\left.25 \mathrm{~s}\right)$ followed by subsequent baking (same parameters as before) and rinsing in developer and methanol. Accordingly, ribs of $20,50,100,200$ and $400 \mu \mathrm{m}$ width, $800 \mathrm{~nm}$ height and $8 \mathrm{~mm}$ length were developed, (see Figure 1).

Toluene solutions of conjugated polymers were spin-coated at $2500 \mathrm{rpm}$ on top of SU8 ribs. The polymer concentration was adjusted for each material in order to yield approximately $100 \mathrm{~nm}$ thickness. Profilometry performed on coated waveguides indicated similar homogeneity and topography than uncoated ones, (not shown). The chemical structures of polyfluorene derivatives employed are shown in Figure 2(a). Poly(9,9-dioctlyfluorene) (PFO) was purchased from American Dye Source whereas PODPF, $\mathrm{PCzODPF}$, and $\mathrm{Ph}-\mathrm{Cz}-\mathrm{FOH}$ were synthesized elsewhere.[19-20] The set-up used for optical characterization of the waveguides comprised a $\mathrm{Nd}$ :YAG laser ( $355 \mathrm{~nm}$ ) with 300 ps temporal width, and $23 \mathrm{~Hz}$ repetition rate as excitation (a)

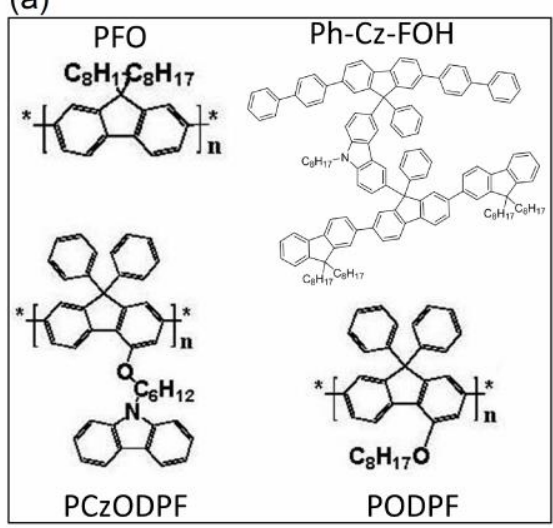

(b)

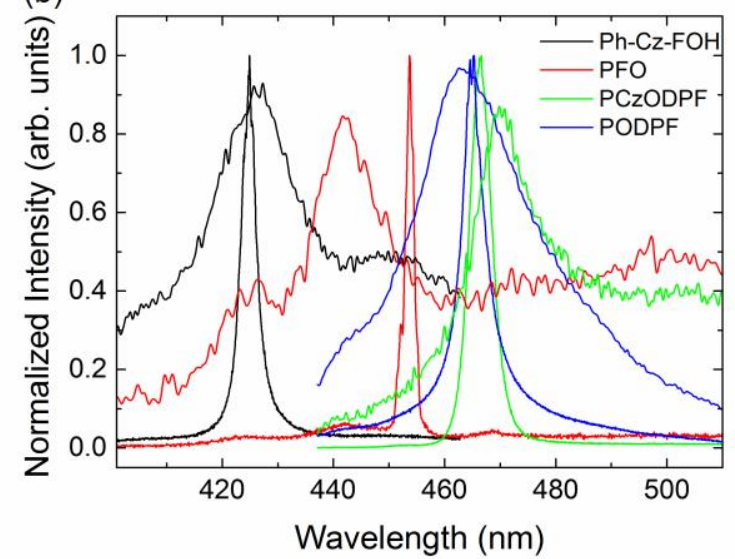

FIGURE 2. (a) Chemical structures of the conjugated polymers used in this study. (b) Corresponding ASE and PL spectra of conjugated polymers deposited on SU8 ribs. 
source. The laser beam was focused with a 100 $\mathrm{mm}$ focal length cylindrical lens to achieve a narrow stripe beam, $(3 \mathrm{~mm}$ long and $40 \mu \mathrm{m}$ width). The length of the beam was adjusted using a tunable slit. Photoluminescence guided along the rib was collected at the edge and focused in free-space configuration on a SP2558 Princeton Instruments (Acton Research) spectrometer equipped with a liquid $\mathrm{N}_{2}$ cooled deep-depleted CCD. Excitation fluence was modified with a set of neutral density filters. Losses were calculated upon moving the excitation stripe along the rib and detecting the Amplified Spontaneous Emission (ASE) spectrum for each position. Threshold values were instead calculated from the dependencies of FWHM and output intensity with fluence, being the final result and average between both values. Final losses and threshold values were the result of averaging among 3-4 analogous waveguides (same width and same conjugated compound). Measurements were carried out in air at room temperature. The topographies of waveguides were visualized with an Alpha Step 120 D Tencor profilometer, able to realize 1D and 2D measurements as well as 3D reconstructions.

\section{RESULTS AND DISCUSSION}

The emission spectra of conjugated polymers are shown in Figure 2(b). PFO is a widely characterized blue emitting polymer whereas Ph-Cz-FOH, PODPF and PCzODPF are novel polyfluorene compounds with emission in the deep blue spectral region. These four compounds exhibit ASE on rib waveguides upon pumping with high fluences, being their ASE peaks located at 454, 425, 465 and $467 \mathrm{~nm}$ respectively. The spectral location of the ASE peaks is in correspondence with the 0-2 photoluminescence (PL) vibronic peak for the four compounds, thus suggesting that they behave as a four-level amplifying medium, in agreement with previous findings in conjugated polymer spincoated films.[21] The $\mathbf{3 0 0}$ ps pulse duration employed as excitation source is close to the PL lifetime values of most the compounds employed in this work.[][][] Thus, ASE is likely to arise from a population inversion build-up involving generation and recombination rates, rather than being of impulsive nature. Figure 3 displays the Output.vs.Input characteristics of four materials on $200 \mu \mathrm{m}$ width ribs. In all cases a sudden drop in the FWHM of emission accompanied by a dramatic emission enhancement with increasing fluence is observed. Note that a collapse of FWHM in the $10^{-4}$ fluence range is observed for $\mathrm{Ph}-\mathrm{Cz}-\mathrm{FOH}$ in contrast with $10^{-2}$ $10^{-1}$ for the other three compounds, thus anticipating superior amplifying performance of the former compound. Figure 4 displays the dependence of the ASE threshold values on waveguide width for the four polymers. In order to understand the effect of rib confinement on ASE threshold we also performed control measurements in conjugated polymers spincoated on top of SU8 films. In all cases, ASE values in ribs were considerably lower. If we focus on PFO for instance we observed almost a nine-fold decrease in ASE threshold when coated on $100 \mu \mathrm{m}$ ribs (from $0.09 \mathrm{mJcm}^{-2}$ in SU 8 film to $0.013 \mathrm{mJcm}^{-2}$ on ribs). 

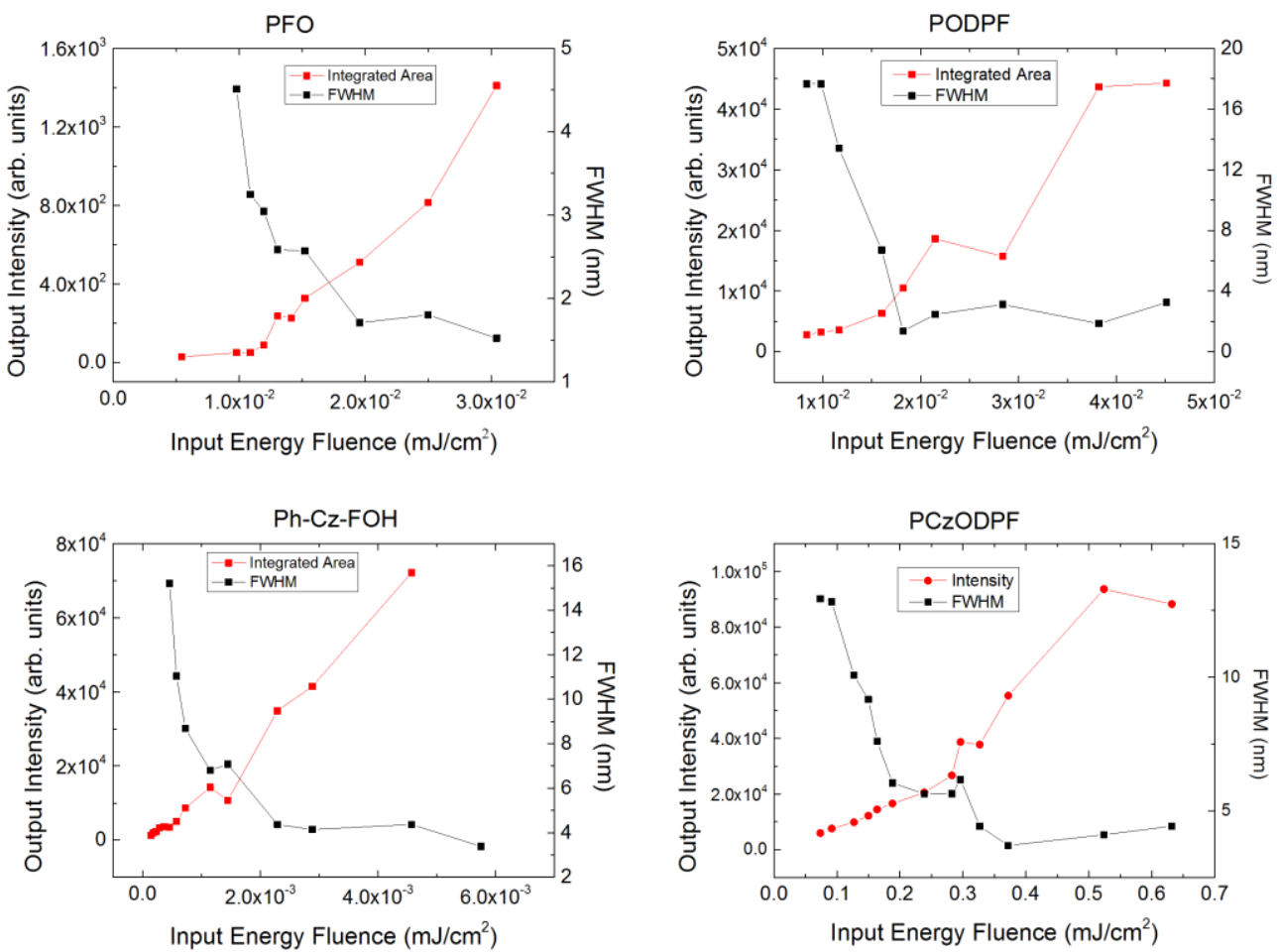

FIGURE 3. Examples of the Input - Output emission characteristics of conjugated polymers on $200 \mu \mathrm{m}$ wide ribs displaying intensity and FWHM as function of fluence.

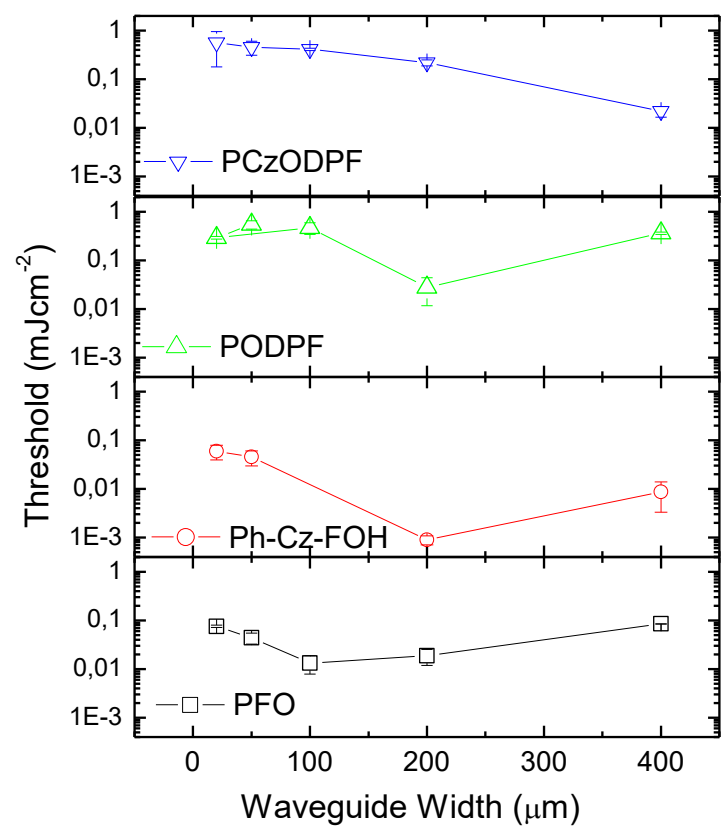

FIGURE 4. ASE threshold values for different polymers and different rib widths. The error bars were obtained from the standard deviation resulting from values measured on four different waveguides.

On account of the highly asymmetric distribution of the fundamental mode in ribs displayed in Figure 1(c), we infer that ASE efficiency enhancement is associated to inplane confinement of the emission induced by the rib geometry, rather than to a displacement of the TEO mode from the substrate to the polymer film. This second strategy has been previously adopted by other authors in symmetric waveguides.[][] Concerning the width dependence of ASE, at first glance the ASE threshold values diminish upon decreasing the widths down to 100 - 200 $\mu \mathrm{m}$ in all cases except for PCzDOPF. This result was reproduced over time and we attribute it to 
the low solubility of this latter compound in toluene and the interplay of aggregate-induced scattering leading to poor amplifying properties of the same. As the rib width gets narrower (50 and $20 \mu \mathrm{m}$ ) the limited resolution at the edges induced by the contact with the shadow mask becomes more important, leading to an overall decrease in planarity of the rib surface. Under these circumstances, larger inhomogeneity of conjugated polymer films deposited on top is expected which can explain the increase in threshold on waveguides with the lowest widths. Comparing the four materials, $\mathrm{Ph}-\mathrm{Cz}-$ $\mathrm{FOH}$ displays the lower thresholds, with a lowest value of $8.910^{-4} \mathrm{mJcm}^{-2}$ found on 200 $\mu \mathrm{m}$ width ribs. This value is more than one order of magnitude lower than that reported by Qian et al. in spin-coated films on top of quartz substrates, $\left(8.3 \quad 10^{-3} \quad \mathrm{mJcm}^{-2}\right) \cdot[19] \quad$ The outstanding optical amplification properties of $\mathrm{Ph}-\mathrm{Cz}-\mathrm{FOH}$ likely arise from the bulky and rigid nature of its carbazole-diarylfluorene side-chain substituents, leading to large steric hindrance and reduced inter-chain interaction. Accordingly, detrimental effects such as aggregation [16] as well as absorption from excited states stabilized between two polymer chains [22] would be partially hindered. Figure 5(a) depicts the dependence of ASE intensity as a function of distance between the excitation stripe and the waveguide edge on a $\mathrm{Ph}-\mathrm{Cz}-\mathrm{FOH}$ $200 \mu \mathrm{m}$ rib. From the exponential dependence we extract a loss coefficient value of $15 \mathrm{~cm}^{-1}$. Comparison between loss coefficients found in waveguides with different widths reflects again slightly upper values for 50 and $20 \mu \mathrm{m}$ ribs (26 and $31 \mathrm{~cm}^{-1}$ respectively), in line with the upper threshold values already shown for same widths. The use of rib waveguides with very low threshold associated allows their ASE operation under low pumping energy regimes.
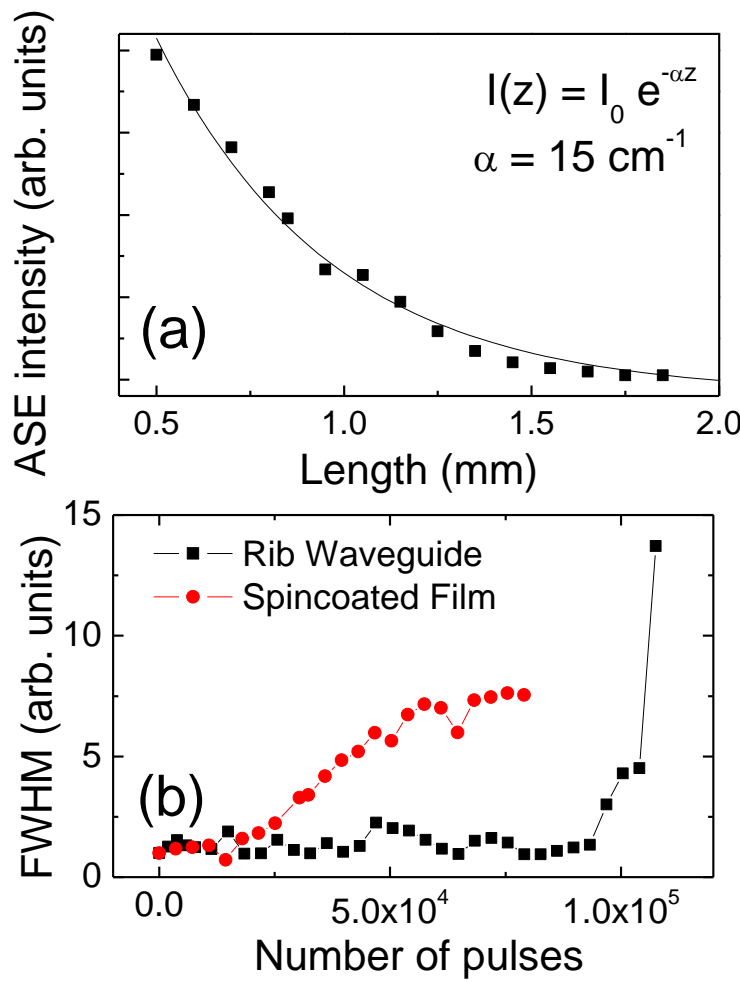

FIGURE 5. (a) Dependence of ASE on distance between stripe and waveguide edge in a 200 $\mu \mathrm{m} \mathrm{Ph}-\mathrm{Cz}-\mathrm{FOH}$ rib depicting an exponential type behavior. (b) Evolution of FWHM of PFO on a $100 \mu \mathrm{m}$ rib upon continuous pulse irradiation.

This is of particular importance since one of the main drawbacks of PFO based optical amplifiers is their poor environmental stability. In order to illustrate this, we compared the ASE degradation of PFO on SU8 rib and film respectively upon continuous photoexcitation. For each sample we tune the pump fluence just above their ASE threshold, $\left(1.09\right.$ and $0.26 \mathrm{mJcm}^{-}$ ${ }^{2}$ for film and waveguide rib respectively), and photoexcite continuously for several minutes recording one spectrum every 1-2 minutes. Figure 5(b) shows the evolution of FWHM upon continuous pulse excitation. For comparative purposes, normalization to the initial values is shown. The FWHM of a PFO film starts to evolve already after photoexcitation with $210^{4}$ pulses. PFO photo-oxidation leads to formation 
of keto defects which act as antennae, funneling PFO singlet excitons and causing a change in the amplifying exciton density. Furthermore, it has been suggested that additional pitfuls arise from losses of waveguiding properties associated to keto defect re-absorption.[Anni] As photo-oxidation becomes more severe the ratio of $\mathrm{PL}$ to $\mathrm{ASE}$ increases leading to a rise in FWHM. Conversely, the FWHM of PFO rib waveguide remains nearly unchanged upon photoexcitation with $910^{4}$ pulses, implying that a much lower photodamage is produced owing to the much lower density of incident photons required for ASE. These observations indicate that the operation lifetime of conjugated polymer amplifiers can be enhanced not only by promoting polymer robustness against photooxidation by synthetic chemistry routes, but also upon appropriate waveguide optimization.

\section{CONCLUSIONS}

Fluorene-based rib waveguides with very low ASE thresholds were developed. Our work suggests that the rib confinement enhances ASE efficiency up to a certain width in the 100-200 $\mu \mathrm{m}$ range. Waveguiding losses on sub $100 \mu \mathrm{m}$ width ribs are likely influenced by the mask contact and shadow effects induced during the photolithography process. We demonstrate that the low ASE threshold values of rib waveguides implies a significant enhancement in long term ASE operation under continuous pulse excitation.

\section{ACKNOWLEDGEMENTS}

G.D.P and J.C-G are grateful for financial support to the Spanish Ministry of Industry and Competiveness through TEC2010-21830-C02-02 project application. N.B. acknowledges financial support through Project Number POIG.01.03.01-14-016/08 (Poland). J.M.O. and
X.Q. acknowledge financial support through project TEC2013-47342-C2 and the European COST Action IC1208. R.X. thanks the National Natural Science Foundation of China (Grants 61376023 and 61136003 ) for financial support,

\section{REFERENCES AND NOTES}

1. A. Rose, Z. Zhu, C. F. Madigan, T. M. Swager, V. Bulović, Nature 2005, 434, 876-879.

2. M. D. McGehee, A. J. Heeger, Adv. Mater. 2000, 12, 1655-1668.

3. J. Cabanillas-Gonzalez, G. Grancini, G. Lanzani, Adv. Mater. 2011, 23, 54685485.

4. F. Hide, M. A. Díaz-García, B. J. Schwartz, M. R. Andersson, Q. Pei, A. Heeger, Science 1996, 273, 1833-1836.

5. I. D. W. Samuel, G. A. Turnbull, Chem. Rev. 2007, 107, 1272-1295.

6. C. Rothe, F. Galbrecht, U. Scherf, A. Monkman, Adv. Mater. 2006, 18, 21372140.

7. S. Zulkarnaen Bisri, T. Takenobu, Y. Iwasa, J. Mater. Chem. C 2014, 2, 28272836.

8. G. Heliotis, D. D. C. Bradley, G. A. Turnbull, I. D. W. Samuel, App. Phys. Lett. 2002, 81, 415-417.

9. T. Virgili, J. Clark, J. Cabanillas-Gonzalez, L. Bazzana, K. C. Vishnubhatla, R. Osellame, R. Ramponi, G. Lanzani, J. Mater. Chem. 2010, 20, 519-523.

10. A. Charas, A. L. Mendonça, J. Clark, J. Cabanillas-Gonzalez, L. Bazzana, A. Nocivelli, G. Lanzani, J. Morgado, Front. Optoelectron. China 2010, 3, 45-53.

11. M. Ariu, D. G. Lidzey, M. Sims, A. J. Cadby, P. A. Lane, D. D. C. Bradley, J. Phys: Condens. Matter 2002, 14, 99759986.

12. M. Campoy-Quiles, G. Heliotis, R. Xia, M. Ariu, M. Pintani, P. Etchegoin, D. D. C. Bradley, Adv. Func. Mater. 2005, 15, 925-933.

13. U. Scherf, E.J.W. List, Adv. Mater. 2002, 14, 477-487. 
14. M. J. Frampton, H. L. Anderson, Angew. Chem. Int. Ed. 2007 , 46, 1028.

15. M. M. Mróz, G. Sforazzini, Y. Zhong, K. S. Wong, H. L. Anderson, G. Lanzani, J. Cabanillas-Gonzalez, Adv. Mater. 2013, 25, 4347-4351.

16. S. W. Thomas III, G. D. Joly, T. M. Swager, Chem. Rev. 2007, 107, 13391386.

17. D. Sahoo, K. Sugiyasu, Y. Tian, M. Takeuchi, I. G. Scheblykin, Chem. Mater. 2014, 26, 4867-4875.

18. J. Cabanillas-Gonzalez, C. Sciascia, G. Lanzani, S. Toffanin, R. Capelli, M. C. Ramon, M. Muccini, J. Gierschner, T-Y Hwu, K-T Wong, J. Phys. Chem B 2008, 112, 11605-11609.
19. Y. Qian, Q. Wei, G. Del Pozo, M. M. Mróz, L. Lüer, S. Casado, J. CabanillasGonzalez, Q. Zhang, L. Xie, R. Xia, W. Huang, Adv. Mater. 2014, 26, 29372942.

20. J. Lin, Z. Lin, B. Liu, Z. Lei, G. del Pozo, J. Cabanillas-Gonzalez, X. Zhang, L. Xie, G. Xing, D. Qiu, R. Xia, W. Huang, submitted.

21. M. Zavelani-Rossi, S. Perissinotto, G. Lanzani, M. Salerno, G. Gigli, Appl. Phys. Lett. 2006, 89, 181105.

22. T. Virgili, D. Marinotto, C. Manzoni, G. Cerullo, and G. Lanzani, Phys. Rev. Lett. 2005, 94, 117402. 


\section{GRAPHICAL ABSTRACT}

Gonzalo Del Pozo', Noureddine Bennis ${ }^{2}$, Xabier Quintana ${ }^{3}$, Jose Manuel Otón ${ }^{3}$, Jinyi Lin ${ }^{4}, Q i$ Wei $^{4}$ Ruidong $\mathrm{Xia}^{4 *}$, Ramon Bernardo ${ }^{1}$, Daniel Granados $^{1}$ and Juan Cabanillas-Gonzalez ${ }^{1 *}$

Fluorene-Based Rib Waveguides with Optimized Geometry for Long-Term Amplified Spontaneous Emission Stability

Long-term operation of blue emitting polyfluorene-based optical amplifiers is compromised by their relatively low photostability. In this work we develop rib waveguides based on different fluorene-based compounds with different side-chain substituents. We observe a nine-fold reduction in energy threshold values for amplified spontaneous emission respect to thin films which is associated to confinement and enhanced waveguiding properties. We demonstrate how appropriate rib width optimization allows for a significant enhancement in amplified spontaneous emission stability during continuous pulsed excitation.

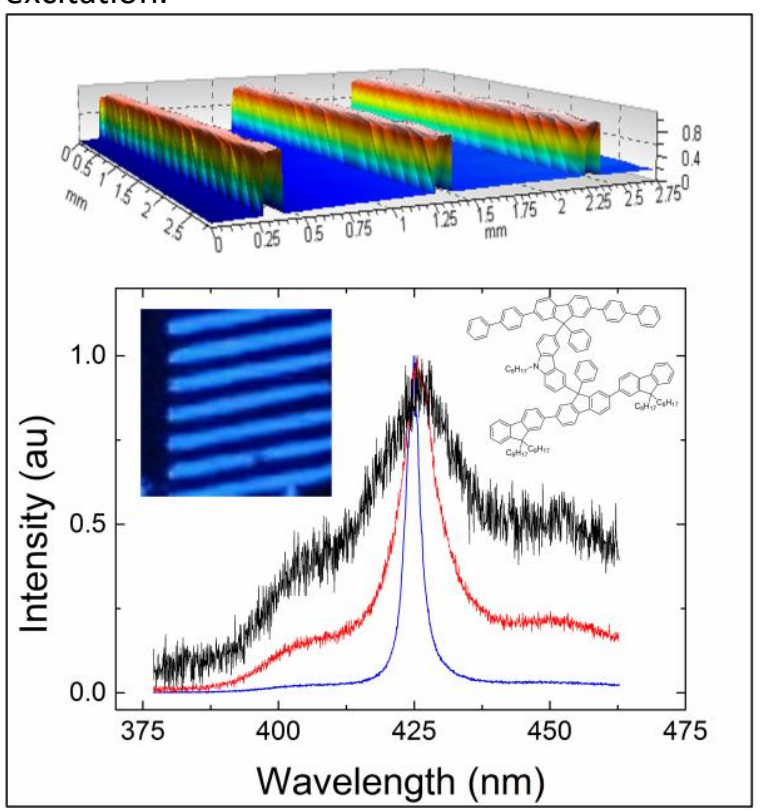

